\title{
New Aspects on Square Roots of a Real $2 \times 2$ Matrix and Their Geometric Applications
}

\author{
Mircea Crasmareanu* and Andrei Plugariu
}

(Communicated by Cihan ÖZGÜR)

\begin{abstract}
We present a new study on the square roots of real $2 \times 2$ matrices with a special view towards examples, some of them inspired by geometry.
\end{abstract}

Keywords: real $2 \times 2$ matrix; square root.

AMS Subject Classification (2010): Primary: 15A24 ; Secondary: 15A18.

${ }^{*}$ Corresponding author

We begin with the following general matrix $A=\left(\begin{array}{ll}a & c \\ b & d\end{array}\right) \in M_{2}(\mathbb{R})$ and ask: is there a matrix $B \in M_{2}(\mathbb{R})$ such that $B^{2}=A$ ? Such a matrix $B$ is called square root of $A$. We point out that the more complicated case of a real matrix of order 3 is discussed in [4]. Although the case we consider is also well studied (according to the bibliography of [3]) we add several examples and facts concerning this notion as well as a series of geometrical applications.

The Euclidean example We recall the n-orthogonal group: $O(n)=\left\{A \in M_{n}(\mathbb{R}): A^{t} \cdot A=I_{n}\right\}$; is the invariant group of the Euclidean inner product $<\cdot, \cdot>$ (yielding the usual Euclidean norm $\|\cdot\|$ ). If $A \in O(n)$ then $\left(\operatorname{det} A^{t}\right) \cdot(\operatorname{det} A)=\operatorname{det} I_{n}=1 \mathrm{implies}$ that $\operatorname{det} A= \pm 1$. Hence, the orthogonal group splits into two components:

$$
O(n)=S O(n) \sqcup O^{-}(n)
$$

where $S O(n)$ contains the matrices from $O(n)$ having $\operatorname{det} A=1$ and $O^{-}(n)$ those with $\operatorname{det} A=-1$; $\sqcup$ represents the disjoint reunion of sets. $S O(n)$ is a subgroup in $O(n)$ and is called $n$-special orthogonal group. $O^{-}(n)$ is not closed under product: $A_{1}, A_{2} \in O^{-}(n)$ implies that $A_{1} A_{2} \in S O(n)$.

Since $M_{1}(\mathbb{R})=\mathbb{R}$ we have that $O(1)=\{ \pm 1\}$ with $S O(1)=\{1\}$ and $O^{-}(1)=\{-1\}$; we remark that $O(1)$ contains the integer unit roots! We know $O(2)$ as well:

$$
R(t)=\left(\begin{array}{cc}
\cos t & -\sin t \\
\sin t & \cos t
\end{array}\right) \in S O(2), \quad S(t)=\left(\begin{array}{cc}
\cos t & \sin t \\
\sin t & -\cos t
\end{array}\right) \in O^{-}(2), \quad t \in \mathbb{R} .
$$

Hence, we have that:

$$
S(t)^{2}=\left(\begin{array}{cc}
\cos t & \sin t \\
\sin t & -\cos t
\end{array}\right)\left(\begin{array}{cc}
\cos t & \sin t \\
\sin t & -\cos t
\end{array}\right)=I_{2}
$$

which means that any $S(t)$ is a root of the unit matrix $I_{2}$. We recall that from a geometrical point of view a square root of the unit matrix is called almost product structure, see for example [6].

Geometrical significance: $R(t)$ is the matrix of rotation of angle $t$ in trigonometrical sense (i.e anticlockwise) around the origin and $S(t)$ is the matrix of axial symmetry with respect to $d_{t / 2}=$ line from plane $\mathbb{R}^{2}$ which contains the origin $O$ and makes the oriented angle $t / 2$ with $O x$. We have that $S\left(t_{2}\right) \cdot S\left(t_{1}\right)=A\left(t_{2}-t_{1}\right) \neq S\left(t_{1}\right) \cdot S\left(t_{2}\right)$.

Received : 09-11-2017, Accepted : 11-01-2018 
We return to the general case of matrix $A$. We remind that $A$ has two invariants:

$$
\operatorname{Tr} A:=a+d, \quad \operatorname{det} A:=a d-b c .
$$

Properties:

i) $\operatorname{Tr}: M_{2}(\mathbb{R}) \rightarrow \mathbb{R}$ is a linear operator: $\operatorname{Tr}\left(\alpha A_{1}+\beta A_{2}\right)=\alpha \operatorname{Tr} A_{1}+\beta \operatorname{Tr} A_{2}$,

ii) det : $M_{2}(\mathbb{R}) \rightarrow \mathbb{R}$ is a multiplicative function: $\operatorname{det}\left(A_{1} A_{2}\right)=\operatorname{det} A_{1} \operatorname{det} A_{2}$,

iii) The characteristic equation of $A$ :

$$
A^{2}-\operatorname{Tr} A \cdot A+\operatorname{det} A \cdot I_{2}=O_{2} .
$$

The multiplicative property of the determinant yields:

The necessary condition for existence of square roots:

$$
\exists B: B^{2}=A \Rightarrow \operatorname{det} A \geq 0 .
$$

Hence we assume from now on that $\operatorname{det} A \geq 0$.

Revised Euclidean example 1: $\operatorname{Tr} S(t)=0, \operatorname{det} S(t)=-1$ which says that $S(t)$ does not admit roots. A root of order 4 of unit matrix is called structure of electromagnetic type according to [7, p. 3807].

We also have relationships between the invariants of $A$ and $B$ :

$$
\operatorname{Tr} A=(\operatorname{Tr} B)^{2}-2 \operatorname{det} B, \quad \operatorname{det} A=(\operatorname{det} B)^{2} .
$$

Proof. It is enough to proof the first identity. We write the characteristic equation of $B$ :

$$
A-\operatorname{Tr} B \cdot B+\operatorname{det} B \cdot I_{2}=O_{2}
$$

which gives:

$$
A=\operatorname{Tr} B \cdot B-\operatorname{det} B \cdot I_{2} .
$$

We square this relation:

$$
A^{2}=(\operatorname{Tr} B)^{2} \cdot A-2 \operatorname{Tr} B \cdot \operatorname{det} B \cdot B+(\operatorname{det} B)^{2} I_{2}
$$

or:

$$
A^{2}-(\operatorname{Tr} B)^{2} \cdot A+2 \operatorname{det} B[\operatorname{Tr} B \cdot B]-(\operatorname{det} B)^{2} I_{2}=O_{2} .
$$

From (1) we have that:

$$
\operatorname{Tr} B \cdot B=A+\operatorname{det} B \cdot I_{2}
$$

which is replaced in square brackets from (3):

$$
A^{2}-(\operatorname{Tr} B)^{2} \cdot A+2 \operatorname{det} B\left[A+\operatorname{det} B \cdot I_{2}\right]-(\operatorname{det} B)^{2} I_{2}=A^{2}-\left[(\operatorname{Tr} B)^{2}-2 \operatorname{det} B\right] \cdot A+(\operatorname{det} B)^{2} I_{2}=O_{2}
$$

and by comparing with the characteristic equation of $A$ we obtain the conclusion.

The relation (4) is fundamental for finding $B$ and we have two cases:

Case I): $\operatorname{Tr} B=0$ implies that: $A=-\operatorname{det} B \cdot I_{2}$.

Case II) $\operatorname{Tr} B \neq 0$ implies that:

$$
B=\frac{1}{\operatorname{Tr} B}\left[A+\operatorname{det} B \cdot I_{2}\right] .
$$

From the first relation (0) we have that:

$$
(\operatorname{Tr} B)^{2}=\operatorname{Tr} A+2|\sqrt{\operatorname{det} A}|
$$

hence, if $A \neq a I_{2}$, we obtain that:

II1) $\operatorname{Tr} A+2 \sqrt{\operatorname{det} A} \leq 0$ implies that $A$ does not have roots,

II2) $\operatorname{Tr} A+2 \sqrt{\operatorname{det} A}>0$ but $\operatorname{Tr} A-2 \sqrt{\operatorname{det} A} \leq 0$ implies that $A$ has two roots:

$$
B_{ \pm}= \pm \frac{1}{\sqrt{\operatorname{Tr} A+2 \sqrt{\operatorname{det} A}}}\left[A+\sqrt{\operatorname{det} A} I_{2}\right]
$$


II3) $\operatorname{Tr} A-2 \sqrt{\operatorname{det} A}>0$ (which implies that $\operatorname{Tr} A+2 \sqrt{\operatorname{det} A}>0$ ) implies that $A$ has four roots:

$$
B_{ \pm}(\varepsilon)= \pm \frac{1}{\sqrt{\operatorname{Tr} A+2 \varepsilon \sqrt{\operatorname{det} A}}}\left[A+\varepsilon \sqrt{\operatorname{det} A} I_{2}\right], \quad \varepsilon= \pm 1 .
$$

Revised Euclidean example 2 For $A(t)$ we have:

$$
\operatorname{Tr} R(t)=2 \cos t, \operatorname{det} R(t)=1, \operatorname{Tr} R(t)+2 \sqrt{\operatorname{det} R(t)}=4 \cos ^{2} \frac{t}{2}, \operatorname{Tr} R(t)-2 \sqrt{\operatorname{det} R(t)}=2(\cos t-1) \leq 0 .
$$

From Case II2, we get that $R(t)$ has two roots:

$$
B_{ \pm}(t)= \pm \frac{1}{2 \cos \frac{t}{2}}\left(\begin{array}{cc}
\cos t+1 & -\sin t \\
\sin t & \cos t+1
\end{array}\right)= \pm R\left(\frac{t}{2}\right)
$$

The relation (10) can be considered the matrix version of the well-known Moivre's relation from complex algebra $(\mathbb{C},+, \cdot)$ :

$$
(\cos t+i \sin t)^{2}=\cos (2 t)+i \sin (2 t)
$$

The group law of $S O(2)$ is: $R\left(t_{1}\right) \cdot R\left(t_{2}\right)=R\left(t_{1}+t_{2}\right)=R\left(t_{2}\right) \cdot R\left(t_{1}\right)$ which gives: $R(t)^{2}=R(2 t)$ and the fact that $S O(2)$ is a group isomorphic to the multiplicative group $\left(S^{1}, \cdot\right)$ of all unit complex numbers.

Inspired by characteristic equation of $A$ we introduce the characteristic polynomial of $A$, namely $p_{A} \in \mathbb{R}[X]$ :

$$
p_{A}(X)=X^{2}-\operatorname{Tr} A \cdot X+\operatorname{det} A .
$$

We know that the possible real roots of $p_{A}$ are called eigenvalues of $A$ and are useful in studying the diagonalisation of $A$. So, if the eigenvalues exist and are different, we denote them by $\lambda_{1}<\lambda_{2}$ and it follows that $A$ admits $a$ diagonal form:

$$
A=S^{-1} \operatorname{diag}\left(\lambda_{1}, \lambda_{2}\right) S
$$

with $S \in G L(2, \mathbb{R})=2$-general linear group i.e. the group of all real invertible matrices of order 2 . Obviously, the condition of existence and inequality for $\lambda_{1,2}$ holds when the discriminant $\Delta\left(p_{A}\right)$ is strictly positive:

$$
\Delta\left(p_{A}\right):=(\operatorname{Tr} A)^{2}-4 \operatorname{det} A .
$$

The relationship between $\Delta\left(p_{A}\right)$ and $\Delta\left(p_{B}\right)$ is given by:

Proposition Let $B$ be a square root of $A$. Then:

$$
\Delta\left(p_{A}\right)=(\operatorname{Tr} B)^{2} \Delta\left(p_{B}\right) .
$$

Thus, if $\operatorname{Tr} B \neq 0$ then $A$ has different eigenvalues if and only if $B$ has different eigenvalues.

Proof. The relation (15) is a direct consequence of $(0)$.

Corollary Suppose the matrix $A$ with $\operatorname{det} A>0$ has the root $B$ with $\operatorname{Tr} B \neq 0$. Assume that $A$ is diagonalisable with $S \in G L(2, \mathbb{R})$ and different eigenvalues $\lambda_{1}<\lambda_{2}$. Then, $0<\lambda_{1}<\lambda_{2}$ and $B$ is diagonalisable with the same matrix $S$ having the eigenvalues $\left\{\sqrt{\lambda_{1}}, \sqrt{\lambda_{2}}\right\}$ or $\left\{-\sqrt{\lambda_{1}},-\sqrt{\lambda_{2}}\right\}$ or $\left\{-\sqrt{\lambda_{1}}, \sqrt{\lambda_{2}}\right\}$ or $\left\{\sqrt{\lambda_{1}},-\sqrt{\lambda_{2}}\right\}$. Equivalently, we are in case II3 with:

$$
B_{ \pm}(\varepsilon)= \pm \frac{1}{\sqrt{\lambda_{2}}+\varepsilon \sqrt{\lambda_{1}}}\left[A+\varepsilon \sqrt{\lambda_{1} \lambda_{2}} I_{2}\right]=S \cdot \operatorname{diag}\left( \pm \sqrt{\lambda_{1}}, \pm \sqrt{\lambda_{2}}\right) \cdot S^{-1} .
$$

Proof. Because $\operatorname{det} A>0$ we have that $\lambda_{1}$ and $\lambda_{2}$ have the same sign. We suppose that $\lambda_{1}<\lambda_{2}<0$. From (6) we have that $(\operatorname{Tr} B)^{2}=\lambda_{1}+\lambda_{2} \pm 2 \sqrt{\lambda_{1} \lambda_{2}}>0$. It follows only the case with + i.e. $-\left|\lambda_{1}\right|-\left|\lambda_{2}\right|+2 \sqrt{\left|\lambda_{1}\right|\left|\lambda_{2}\right|}>0$ but this is impossible because of the AM-GM inequality. Recall that the AM-GM inequality states that the arithmetic mean of a list of non-negative real numbers is greater than or equal to the geometric mean of the same list. 
The golden example It is known that the golden proportion (or the golden number) is the positive root, $\phi=\frac{\sqrt{5}+1}{2}$, of the equation [6]:

$$
x^{2}-x-1=0 .
$$

The negative root is $-\phi^{-1}=\frac{1-\sqrt{5}}{2}$. Let us consider the matrix:

$$
A=\left(\begin{array}{ll}
3 & 2 \\
2 & 3
\end{array}\right), \quad \operatorname{Tr} A=6, \operatorname{det} A=5 .
$$

$A$ is diagonalisable, being symmetric, with $0<\lambda_{1}=1<\lambda_{2}=5$. We have that:

$$
\operatorname{Tr} A+2 \varepsilon \sqrt{\operatorname{det} A}=6+2 \varepsilon \sqrt{5}=(\sqrt{5}+\varepsilon)^{2}
$$

We are in Case II3 and for example:

$$
B_{ \pm}(1)= \pm \frac{1}{\sqrt{5}+1}\left(\begin{array}{cc}
3+\sqrt{5} & 2 \\
2 & 3+\sqrt{5}
\end{array}\right)= \pm \frac{1}{2 \phi}\left(\begin{array}{cc}
2 \phi^{2} & 2 \\
2 & 2 \phi^{2}
\end{array}\right)= \pm\left(\begin{array}{cc}
\phi & \phi^{-1} \\
\phi^{-1} & \phi
\end{array}\right) .
$$

By analogy with the problem studied here, we call the matrix $A \in M_{n}(\mathbb{R})$ satisfying $A^{2}-A-I_{n}=O_{n}$, as being an almost golden structure. In [6] we study the relationship between almost golden structures and almost product structures.

We return to the case $I$ given by $A=a I_{2}$ and we present the solution from [3, p. 491]. We have, irrespective of $a$ 's sign, an infinity of roots:

$$
B_{ \pm}(c, s):= \pm\left(\begin{array}{cc}
c & s \\
\frac{a-c^{2}}{s} & -c
\end{array}\right), \quad c \in \mathbb{R}, s \in \mathbb{R}^{*} .
$$

If $a=0$ then we add the infinite set of almost tangent structures:

$$
B_{ \pm}(u):= \pm\left(\begin{array}{ll}
0 & 0 \\
u & 0
\end{array}\right), \quad u \in \mathbb{R}
$$

If $a>0$ then we add the infinite set:

$$
B_{ \pm}(u):=\left(\begin{array}{cc} 
\pm \sqrt{a} & 0 \\
u & \mp \sqrt{a}
\end{array}\right), \quad B_{ \pm}:=\left(\begin{array}{cc} 
\pm \sqrt{a} & 0 \\
0 & \pm \sqrt{a}
\end{array}\right) .
$$

Revised Euclidean example 3 For $a=1$ the family $B_{+}(c, s)$ becomes:

$$
B(c, s)=\left(\begin{array}{cc}
c & s \\
\frac{1-c^{2}}{s} & -c
\end{array}\right)
$$

which gives:

$$
B(\cos t, \sin t)=S(t) \text {. }
$$

This way we obtain the matrices from $O^{-}(2)$. We consider now a right triangle with sides $x, y$ and hypotenuse $z$. We have that:

$$
S(t)=\frac{1}{z}\left(\begin{array}{cc}
x & y \\
y & -x
\end{array}\right) .
$$

If $(x, y, z) \in\left(\mathbb{N}^{*}\right)^{3}$ then $(x, y, z)$ is a Pythagorean triple. This example of almost product structures provided by Pythagorean triples appears on the Web page [1]. In [5] we gave a method for finding matrices $A \in M_{3}(\mathbb{R})$ which transforms a Pythagorean triple into another Pythagorean triple.

Open problem Do the matrices $A$ which preserve Pythagorean triples admit roots?

We return now to the given corollary: a symmetric matrix $A$ with different and strictly positive eigenvalues is positive definite, [2]. Thus, $A$ defines a new inner product on $\mathbb{R}^{n}$ :

$$
<\bar{x}, \bar{y}>_{A}:=<\bar{x}, A \bar{y}>\text {. }
$$


If $A$ admits $B$ as a root then:

$$
<\bar{x}, \bar{y}>_{A}:=<\bar{x}, B^{2} \bar{y}>=<B^{t} \bar{x}, B \bar{y}>.
$$

If $B$ is also symmetric, which happens when $n=2$, then:

$$
<\bar{x}, \bar{y}>_{A}:=<B \bar{x}, B \bar{y}>
$$

hence:

$$
\|\bar{x}\|_{A}^{2}=\|B \bar{x}\|^{2} .
$$

Thus, for nonzero vectors $\bar{x}, \bar{y} \in \mathbb{R}^{n}$, the angle $\varphi_{A}(\bar{x}, \bar{y})$ between them with respect to $\langle\cdot, \cdot\rangle_{A}$ is given by:

$$
\cos \varphi_{A}(\bar{x}, \bar{y})=\cos \varphi(B \bar{x}, B \bar{y}) .
$$

Generalized golden example The matrix $A \in M_{2}(\mathbb{R})$ is called bi-symmetric if it has the form:

$$
A=\left(\begin{array}{cc}
a & b \\
b & a
\end{array}\right)
$$

Then $\operatorname{det} A=a^{2}-b^{2}$ and, for having roots, we assume that $a>b$. We are in case II 3 and obtain that:

$$
B_{ \pm}(\varepsilon)= \pm \frac{1}{2}\left(\begin{array}{ll}
\sqrt{a+b}+\varepsilon \sqrt{a-b} & \sqrt{a+b}-\varepsilon \sqrt{a-b} \\
\sqrt{a+b}-\varepsilon \sqrt{a-b} & \sqrt{a+b}+\varepsilon \sqrt{a-b}
\end{array}\right)
$$

which gives us the result that any of its root is also bi-symmetric. The conversely: If $B$ is bi-symmetric then $B^{2}$ is bi-symmetric is obvious from calculus.

Hyperbolic example We consider:

$$
A(t):=\left(\begin{array}{cc}
\cosh t & \sinh t \\
\sinh t & \cosh t
\end{array}\right) .
$$

$A$ is a bi-symmetric matrix with $a>b$ and with formula (33) we obtain that:

$$
B_{ \pm}(1)= \pm\left(\begin{array}{cc}
\cosh \frac{t}{2} & \sinh \frac{t}{2} \\
\sinh \frac{t}{2} & \cosh \frac{t}{2}
\end{array}\right), \quad B_{ \pm}(-1)= \pm\left(\begin{array}{cc}
\sinh \frac{t}{2} & \cosh \frac{t}{2} \\
\cosh \frac{t}{2} & \sinh \frac{t}{2}
\end{array}\right) .
$$

Fibonacci example In [8, p. 24] is introduced the Q-Fibonacci matrix:

$$
Q=\left(\begin{array}{ll}
1 & 1 \\
1 & 0
\end{array}\right)
$$

that has the natural powers:

$$
Q^{n}=\left(\begin{array}{cc}
F_{n+1} & F_{n} \\
F_{n} & F_{n-1}
\end{array}\right) .
$$

Because of the golden example, we consider the matrix:

$$
Q(n)=\left(\begin{array}{cc}
F_{n+1} & F_{n} \\
F_{n} & F_{n+1}
\end{array}\right) .
$$

With relation (33) we have the roots:

$$
Q_{ \pm}(n, \varepsilon)= \pm \frac{1}{2}\left(\begin{array}{cc}
\sqrt{F_{n+2}}+\varepsilon \sqrt{F_{n-2}} & \sqrt{F_{n+2}}-\varepsilon \sqrt{F_{n-2}} \\
\sqrt{F_{n+2}}-\varepsilon \sqrt{F_{n-2}} & \sqrt{F_{n+2}}+\varepsilon \sqrt{F_{n-2}}
\end{array}\right) .
$$

Almost complex example A root of the matrix $-I_{2}$ is called almost complex structure. According to (21) we have:

$$
B_{ \pm}(s, c):= \pm\left(\begin{array}{cc}
s & c \\
\frac{-1-s^{2}}{c} & -s
\end{array}\right), \quad s \in \mathbb{R}, c \in \mathbb{R}^{*} .
$$


An interesting particular case is:

$$
B_{ \pm}(\sinh t, \cosh t):=B(t)= \pm\left(\begin{array}{cc}
\sinh t & \cosh t \\
-\cosh t & -\sinh t
\end{array}\right) .
$$

Acknowledgements The authors are extremely indebted to two anonymous referees for their extremely useful remarks and improvements.

\section{References}

[1] https://en.wikipedia.org/wiki/Square_root_of_a_matrix

[2] https://ro.wikipedia.org/wiki/Positive-definite_matrix

[3] Anghel, N., Square roots of real $2 \times 2$ matrices, Gaz. Mat. Ser. B 118 (2013), no. 11, 489-491.

[4] Anghel, N., Square roots of real 3x3 matrices vs. quartic polynomials with real zero, An. Ştiinţ. Univ. "Ovidius" Constanţa, Ser. Mat. 25 (2017), no. 3, 45-58.

[5] Crasmareanu, M., A new method to obtain Pythagorean triple preserving matrices, Missouri J. Math. Sci. 14 (2002), no. 3, 149-158. MR 1929067(2003h:15041), Zbl 1032.15007

[6] M. Crasmareanu, M. and Hrețcanu, Cristina-Elena, Golden differential geometry, Chaos, Solitons \& Fractals 38 (2008), no. 5, 1229-1238. MR 2456523(2009k:53059)

[7] Reyes, E., Cruceanu, V. and Gadea, P. M., Structures of electromagnetic type on vector bundle, J. Phys. A, Math. Gen. 32 (1999), no. 20, 3805-3814. Zbl 0969.53041

[8] Stakhov A. and Aranson S., The "golden" non-Euclidean geometry. Hilbert's fourth problem, "golden" dynamical systems, and the fine-structure constant. With the assistance of Scott Olsen. Series on Analysis, Applications and Computation 7. Hackensack, NJ: World Scientific, 2017. Zbl 1351.51002

\section{Affiliations}

Mircea CRASMAREANU

ADDRESS: University "Al. I.Cuza", Faculty of Mathematics, 700506, Iasi-Romania.

E-MAIL: mcrasm@uaic.ro

ORCID ID: $0000-0002-5230-2751$

ANDREI PLUgariu

ADDRESS: University "Al. I.Cuza", Faculty of Mathematics, 700506, Iasi-Romania.

E-MAIL: plugariu.andrei@yahoo.ro 